\section{An alternative lifestyle for immortalized oral keratinocytes}

\author{
Roger R. Reddel
}

Children's Medical Research Institute, 214 Hawkesbury Road, Westmead, Sydney 2145, Australia. Phone: 61-2-9687-2800; Fax: 61-2-9687-2120; E-mail: rreddel@cmri.usyd.edu.au.

J. Clin. Invest. 108:665-667 (2001).DOI:10.1172/JCI200113818.
Normal human somatic cells divide only a limited number of times before they enter a permanent state of cell cycle arrest referred to as senescence (1). If cells can divide 40 times, it might be expected that they could form a mass containing $2^{40}$ cells, equating to a 1-kg tumor. This simple calculation, however, overlooks the significant rate of intratumoral cell death and the need for a moderately large number of (epi)genetic changes to accumulate lution and result in a fully malignant phenotype. When these factors are taken into account, it seems clear that the number of cell divisions required to form a clinically significant tumor greatly exceeds the proliferative capacity of normal cells (2). Identification of the molecular events involved in breaching the senescence barrier may reveal key targets for novel anticancer drugs. A study of the immortalization of a human cell type relevant to head and neck cancer, reported in this issue of the JCI, adds to the current understanding of this subject (3).

\section{Disruption of tumor suppressor gene functions}

Squamous cell carcinomas of the head and neck frequently exhibit two key genetic alterations: mutation of the p53 gene, and expression of high levels of cyclin D1 (4). Opitz et al. made these alterations in otherwise normal human oral keratinocytes (3), and they found that each change could individually increase the number of times the cells could divide. When combined, these changes had an additive effect, greatly increasing the proliferative capacity of the cells (Figure 1). True immortalization required one or more additional spontaneous changes in the cells and occurred between population doublings 120 and 160 .

The findings regarding these key cancer genes fit very well with other studies on the control of proliferative during successive phases of clonal evo- potential. It has long been known that simian virus 40 (SV40) can extend the proliferative life span of human cells by a finite amount (5). This effect is dependent on the ability of an SV40encoded protein, large $T$ antigen, to disrupt the function of the protein products of two key cellular tumor suppressor genes, p53 and Rb (reviewed in ref. 6). Oncogenic strains of human papillomavirus (HPV) also produce proteins that interfere with the $\mathrm{p} 53$ and $\mathrm{Rb}$ proteins (6). Cells from individuals who inherited a mutant copy of $p 53$ sometimes undergo a spontaneous increase in proliferative potential associated with loss of the wild-type $p 53$ allele, and a further increase associated with loss of p16 ${ }^{\text {INK4a }}$ function $(7,8)$.

A common thread in these and other studies on the control of proliferative potential is the key role of p53 (9). The other proteins, cyclin $\mathrm{D} 1, \mathrm{Rb}$, and $\mathrm{p} 16^{\mathrm{INK} 4 \mathrm{a}}$, are all linked in a common pathway that is disrupted in the great majority of cancers: in its active state $\mathrm{Rb}$ inhibits progression through the cell division cycle; cyclin D1 is part of a complex that inactivates $\mathrm{Rb}$; and $\mathrm{p} 16^{\mathrm{INK} 4 \mathrm{a}}$ inhibits the action of the cyclin D1-containing complex. Disruption of either Rb or p $16^{\mathrm{INK} 4 a}$ function increases prolifera- tive potential (10). It might be expected that cyclin D1 overexpression would have a similar effect, and this has now been demonstrated (3).

\section{Maintenance of telomeres}

Since the disruption of p53 and of the $\mathrm{Rb}$ pathway does not suffice to give cells unlimited proliferative capacity (10), other limitations must exist that are overcome during immortalization. Olovnikov (11) predicted that the chromosome ends (telomeres) of normal somatic cells would undergo shortening with every cell division and that this would limit cell division potential. Telomeres contain repetitive DNA to which specific binding proteins attach and form a protective cap structure. Many studies have confirmed the prediction that the telomeres of normal somatic cells shorten with cell division (11). Nonimmortalized cells expressing the SV40 oncogenes, or with spontaneous loss of $\mathrm{p} 53$ and $\mathrm{p} 16^{\mathrm{INK} 4 \mathrm{a}}$ function, or with mutant p53 plus overexpressed cyclin D1, also undergo progressive telomere shortening $(3,7$, 12). Immortalization is associated with activation of a mechanism that prevents further telomere shortening. This mechanism usually involves telomerase, a ribonucleoprotein complex that synthesizes telomeric DNA by

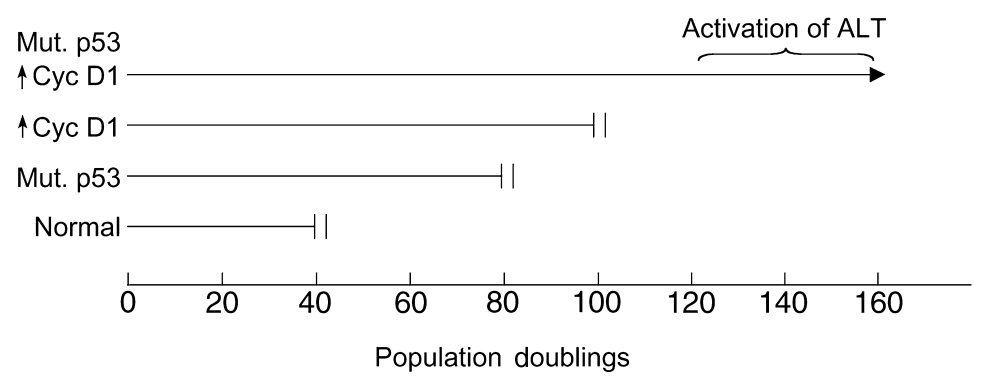

Figure 1

The proliferative potential of oral keratinocytes is extended by mutant p53 or overexpression of cyclin D1. Combining these changes permitted activation of a telomerase-independent mechanism of telomere maintenance (alternative lengthening of telomeres [ALT]) and led to cell immortalization (3). Permanent proliferation arrest (senescence or crisis) is denoted by double vertical bars. 
$\mathbf{a}$
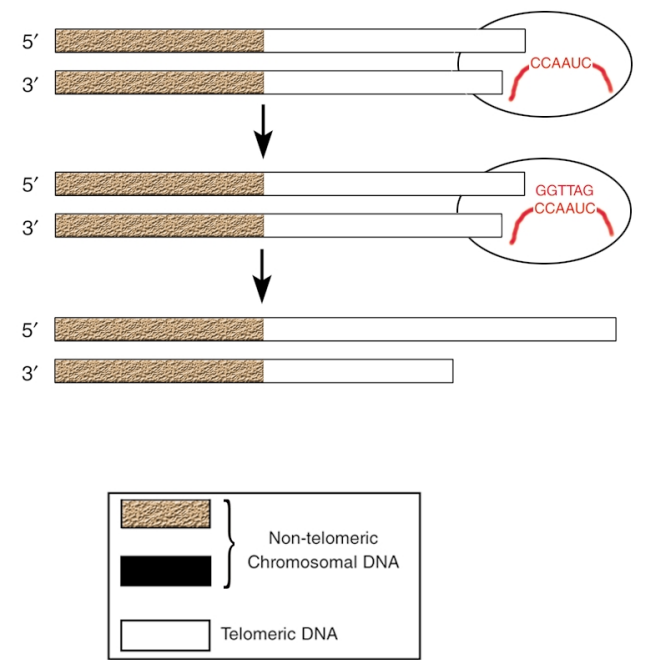

b

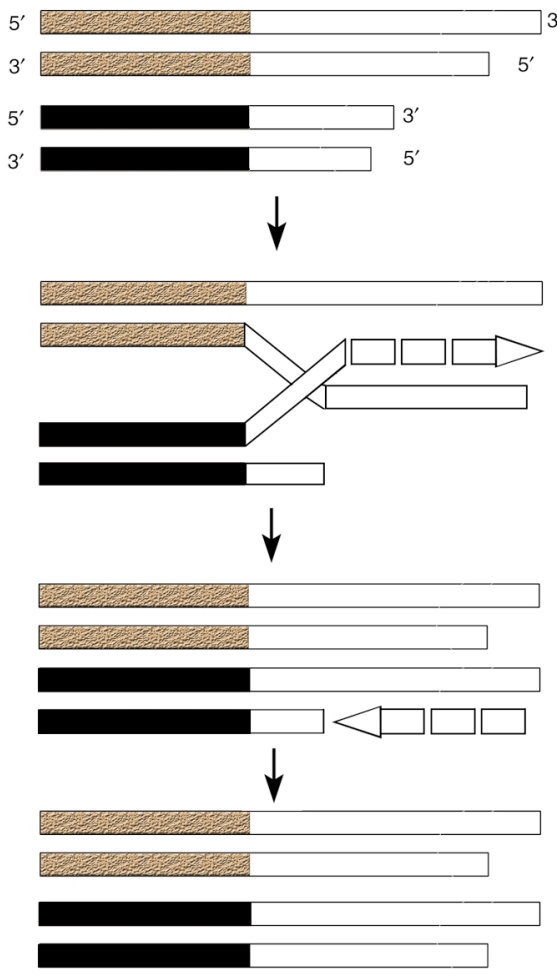

\section{Figure 2}

Mechanisms for the maintenance of telomeres. (a) The enzyme telomerase contains an RNA molecule (shown in red) that it uses as a template to synthesize new telomeric DNA by reverse transcription (13). (b) Telomeres may also be lengthened by an ALT mechanism. All telomeres have the same repetitive sequence, so one telomere can use another telomere as a template for synthesis of telomeric DNA (17). reverse transcription (13). Telomerase is present in most cancers (14), and inhibition of telomerase activity abrogates immortalization and tumorigenicity (15), making telomerase an attractive drug target.

Telomere elongation sometimes occurs in the absence of telomerase, however, by one or more mechanisms known as alternative lengthening of telomeres (ALT). Genetic changes known to be permissive for ALT activation include expression of SV40 or HPV oncogenes, and spontaneous loss of p53 and p16 ${ }^{\mathrm{INK} 4 \mathrm{a}}$ function; these changes can also result in activation of telomerase $(7,8,16)$. Now, Opitz et al. (3) have shown that mutation of p53 coupled with overexpression of cyclin D1 permits activation of ALT. Although it is possible that these changes participate directly in the derepression of ALT (or telomerase), it is very likely that they predispose to immortalization by increasing the probability that additional genetic changes required for activation of ALT (or telomerase) will occur, for example by fostering genetic instability and by expanding the cell population via a finite increase in proliferative potential. The stochastic nature of these additional changes explains why crisis is not always observed: If a telomere maintenance mechanism is activated sufficiently early in one or more cells, their progeny may take over the culture as the other cells cease dividing.

For some ALT cells, it has been demonstrated that the ALT mechanism involves intertelomeric recombination and copy templating for synthesis of telomeric DNA (17). In all of the human ALT cell lines identified to date, telomeres are heterogeneous in length, ranging from extremely short to extremely long (16). Most ALT cells contain a few chromosome ends that appear to be poorly protected by telomere sequences (18), possibly resulting in chromosomal instability. ALT cell lines are also characterized by the presence of extrachromosomal nuclear aggregates of telomeric DNA, specific telomere binding proteins, and proteins involved in recombination (19). The role of these aggregates is unknown, but they are a very useful marker for the presence of ALT.

ALT occurs in human tumors, especially sarcomas (20). ALT and telomerase can coexist in the same cells in culture (18), and in tumors (20). Telomerase inhibitors are likely to be ineffective for tumors that contain ALT-positive cells. It would also be pre- dicted that effective blockade of telomerase activity would exert a potent selection pressure for the activation of ALT in a previously telomerase-positive tumor. Successful telomere-directed therapy may require a combination of telomerase and ALT inhibitors to prevent the emergence of drug resistance.

1. Hayflick, L., and Moorhead, P.S. 1961. The serial cultivation of human diploid cell strains. Exp. Cell Res. 25:585-621.

2. Reddel, R.R. 2000. The role of senescence and immortalization in carcinogenesis. Carcinogenesis. 21:477-484.

3. Opitz, O.G., Suliman, Y., Hahn, W.C., Blum, H.E., and Rustgi, A.K. 2001. Cyclin D1 overexpression and p53 inactivation immortalize primary oral keratinocytes by a telomerase-independent mechanism. J. Clin. Invest. 108:725-732.

4. Bartkova, J., et al. 1995. Abnormal patterns of Dtype cyclin expression and $G_{1}$ regulation in human head and neck cancer. Cancer Res. 55:949-956.

5. Girardi, A.J., Jensen, F.C., and Koprowski, H. 1965 SV40-induced transformation of human diploid cells: crisis and recovery. J. Cell. Physiol. 65:69-84.

6. Bryan, T.M., and Reddel, R.R. 1994. SV40-induced immortalization of human cells. Crit. Rev. Oncog. 5:331-357.

7. Rogan, E.M., et al. 1995. Alterations in p53 and p16 $6^{I N K 4}$ expression and telomere length during spontaneous immortalization of Li-Fraumeni syndrome fibroblasts. Mol. Cell. Biol. 15:4745-4753.

8. Vogt, M., Haggblom, C., Yeargin, J., ChristiansenWeber, T., and Haas, M. 1998. Independent induction of senescence by $p 16^{I N K 4 a}$ and $p 21^{C I P 1}$ in spontaneously immortalized human fibroblasts. Cell Growth Differ. 9:139-146. 
9. Wynford-Thomas, D. 1996. p53: guardian of cellular senescence. J. Pathol. 180:118-121.

10. Huschtscha, L.I., and Reddel, R.R. 1999. p16 ${ }^{\mathrm{INK} 4 \mathrm{a}}$ and the control of cellular proliferative life span. Carcinogenesis. 20:921-926.

11. Olovnikov, A.M. 1996. Telomeres, telomerase and aging: origin of the theory. Exp. Gerontol. 31:443-448.

12. Counter, C.M., et al. 1992. Telomere shortening associated with chromosome instability is arrested in immortal cells which express telomerase activity. EMBOJ. 11:1921-1929.

13. Blackburn, E.H. 1992. Telomerases. Annu. Rev. Biochem. 61:113-129.
14. Shay, J.W., and Bacchetti, S. 1997. A survey of telomerase activity in human cancer. Eur. J. Cancer 33:787-791.

15. Hahn, W.C., et al. 1999. Inhibition of telomerase limits the growth of human cancer cells. Nat. Med. 5:1164-1170.

16. Bryan, T.M., Englezou, A., Gupta, J., Bacchetti, S. and Reddel, R.R. 1995. Telomere elongation in immortal human cells without detectable telomerase activity. EMBO J. 14:4240-4248.

17. Dunham, M.A., Neumann, A.A., Fasching, C.L., and Reddel, R.R. 2000. Telomere maintenance by recombination in human cells. Nat. Genet 26:447-450.
18. Perrem, K., Colgin, L.M., Neumann, A.A., Yeager T.R., and Reddel, R.R. 2001. Coexistence of alternative lengthening of telomeres and telomerase in hTERT-transfected GM847 cells. Mol. Cell. Biol. 21:3862-3875.

19. Yeager, T.R., et al. 1999. Telomerase-negative immortalized human cells contain a novel type of promyelocytic leukemia (PML) body. Cancer Res. 59:4175-4179.

20. Bryan, T.M., Englezou, A., Dalla-Pozza, L., Dunham, M.A., and Reddel, R.R. 1997. Evidence for an alternative mechanism for maintaining telomere length in human tumors and tumor-derived cell lines. Nat. Med. 3:1271-1274. 